Historic, Archive Document

Do not assume content reflects current scientific knowledge, policies, or practices. 


\section{SPRING 1931 \\ BERRY PLANTS}

Prices in Small and Laroge quantities

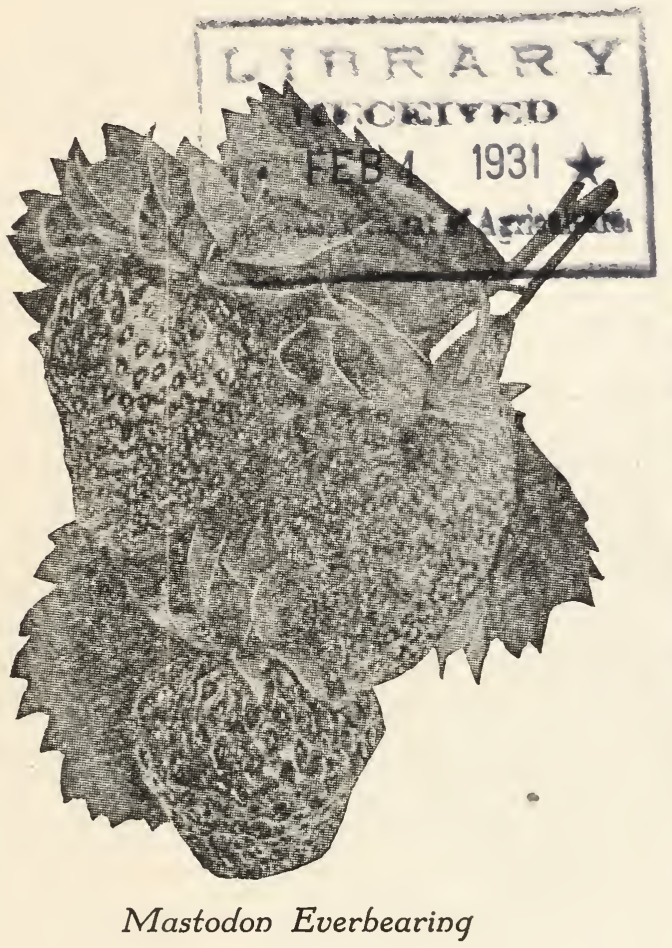

Richards Nursery AND

Berry Gardens

CHINO, CALIFORNIA 


\section{TIME TO PLANT}

Early spring is the most logical, natural, and most successful and satisfactory time for planting all berry plants. December, January and February are best months, whenever the weather permits, and also have the benefit of the rains.

\section{CULTURE METHODS}

We give only general directions for planting of different varieties. Will be pleased to give any information in regards to culture methods.

We do not carry large varieties of berry plants. What we have listed we recommend. We advertise only the best varieties that will bring the best results for commercial and home planting.

\section{$\begin{array}{llllll}\text { P } & \text { R } & \text { I } & \text { C } & \text { E } & \text { S }\end{array}$}

\section{STRA NBERRIES-}

Spring Varieties

$100 \quad 1000$

Blakemore $\$ 2.50 \$ 15.00$

Carolina or Missionary.

Improved Klondike $1.00 \quad 7.00$

Brandywine 1.00

Banner

Everbearing

Måstodon $\$ 2.00$ $\$ 12.00$

Champion $1.50 \quad 10.00$

Progressive $1.50 \quad 10.00$

Write for prices for 5,000 or more.

We recommend in planting spring varieties in rows $2 \frac{1}{2}$ to $3 \frac{1}{2}$ feet apart, plants should be planted in rows from 15 to 24 inches, depending upon the amount of ground you have to spare. Everbearing varieties should be set in row 2 feet apart and 12 inches in the rows. In planting do not cover the crown of the plants with earth, and provision should be made for running irrigation down the rows. Remove all runners at all times unless they are needed to fill rows, or to furnish new plants. Constant irrigation is needed during the growing and bearing season if good berries are to be secured. 


\section{YOUNGBERRIES}

The Youngberry is becoming one of the leading bush berries for commercial and home planting, a very hardy grower, and is more prolific in growing and bearing than any cane berry we have.

The Youngberry is a cross between a Loganberry and Dewberry. Youngberry should be planted from 6 to 8 feet in the rows, and in rows 7 feet apart. If your vines have made vigorous growth it would be advisable to use three wires so as to support the vines and berries.

\section{YOUNGBERRIES-}

$$
\text { Each } 10 \quad 100
$$

$$
\begin{array}{ccrrr}
\text { Transplants-2 } & \text { yr. No.1 } \$ & .40 & \$ 3.00 & \$ 25.00 \\
& \text { No.2 } & .25 & 2.00 & 15.00 \\
\text { Tips-1 yr. ........................... } & & 1.00 & 5.00
\end{array}
$$

Tips-1 yr. Per 1000...........................\$35.00

We also have listed Youngberry transplants, 2 year plants. These plants, if properly taken care of, will bear from 3 to 4 boxes of good berries the first season.

\section{LOGANBERRIES-}

$10100 \quad 1000$

Improved Logan ................\$1.00 \$4.00 \$35.00

\section{DEWBERRIES-}

$10 \quad 100 \quad 1000$

Gardena ...........................\$1.00 \$4.00 $\$ 25.00$

Lucretia

$\begin{array}{lll}1.00 & 4.00 \quad 25.00\end{array}$

Loganberries and Dewberries, which also are trellis growing berries, are not such strong growers as the Youngberry, but do well in most any section, and are very good marketing berries. They should be planted 5 feet in the rows, and in rows 6 feet apart. The bearing wood should be removed each year, soon after the crop is harvested. 


\section{RASPBERRIES-}

$\begin{array}{lll}10 & 100 & 1000\end{array}$

St. Regis Everbearing.......\$ $\$ .50 \quad \$ 3.00 \quad \$ 25.00$

California Surprise .......... $\quad .50 \quad 3.00 \quad 25.00$

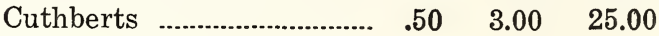

St. Regis Transplants, No. 1

$\begin{array}{ccc}\text { Each } & 10 & 100 \\ .25 & \$ 1.50 & \$ 10.00\end{array}$

Raspberries will do well on almost any soil of reasonable depth. The St. Regis will bear the first year providing they have plenty of water, and do not require the trellising as the Cuthberts or the California Surprise do. They should be trellised after the first year so as to support the vines and crop. Raspberries should be planted about 3 feet apart in the rows and the rows 6 feet apart.

\section{BLACKBERRIES-}

\begin{tabular}{|c|c|c|c|}
\hline & 10 & 100 & 1000 \\
\hline Mammoth Black & $\ldots \$ 1.00$ & $\$ 5.00$ & $\$ 35.00$ \\
\hline Cory Thornless & $\ldots 1.00$ & 5.00 & 35.00 \\
\hline Himalaya ........... & 1.00 & 5.00 & 25.00 \\
\hline Crandle .... & 1.00 & 4.00 & 25.00 \\
\hline Macatawa .......... & $\ldots 1.00$ & 5.00 & 30.00 \\
\hline
\end{tabular}

Blackberries do well in most any soil, the distance in planting varies according to the variety. Himalayas and Mammoth Thornless, which are strong growers, should be spaced at least 8 feet in the rows and rows 7 feet apart. Crandle and Macatawa should be planted about 5 feet in the rows and rows 7 feet apart. Crandle do not require any trellis to grow on, but the Mammoth and Himalaya should be trained by evenly spaced wires running between single stakes.

\section{BLACK CAPS RASPBERRIES-}

$10 \quad 100 \quad 1000$

Cumberland ….....................\$1.00 $\$ 4.00 \quad \$ 25.00$

King of the Cliff................ $1.00 \quad 4.00 \quad 25.00$ 


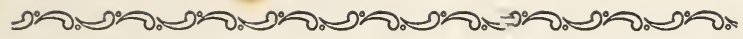

\section{FRUIT AND NUT TREES}

PEACHES-4 to $6 \mathrm{ft}$. Trees-

Each Per 10

Elberta

$\$ .50 \quad \$ 4.00$

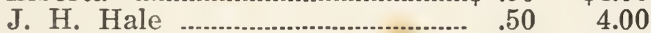

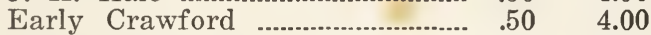

Briggs Red May ............................... $\quad .50 \quad 4.00$

Early Inperial …............................. $\quad .50 \quad 4.00$

Foster …........................................... $\quad .50 \quad 4.00$

George IV _.................................... $\quad .50 \quad 4.00$

Lovell _................................................ $\quad .50 \quad 4.00$

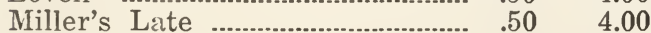

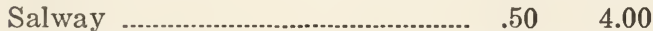

Strawberry Free ……...................... $.50 \quad 4.00$

Lukens Honey …............................. $\quad .50 \quad 4.00$

White Heath Cling .......................... $\quad .50 \quad 4.00$

Orange Cling …….......................... $\quad .50 \quad 4.00$

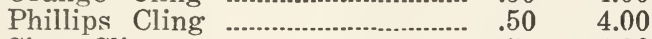

Sims Cling ....................................... $\quad .50 \quad 4.00$

NECTARINES-4 to $6 \mathrm{ft}$.-

Each Per 10

Gower ….................................. \$ $.50 \quad \$ 4.00$

Stanwick .......................................... $\quad .50 \quad 4.00$

FIGS-4 to $6 \mathrm{ft}$.-

Each Per 10

Black Mission …..............................\$ .50 $\$ 4.00$

White Kadota ............................... $\quad .50 \quad 4.00$

Brown Turkey …........................... $\quad .50 \quad 4.00$

APRICOTS-4 to $6 \mathrm{ft}$.-

Each Per 10

Royal Cot .................................. \$ .50 $\$ 4.00$

Early New Castle .......................... $\quad .50 \quad 4.00$

Tilton ….............................................. $\quad .50 \quad 4.00$

Mónepark ……............................. $\quad .50 \quad 4.00$

\section{ALMONDS-}

Each Per 10

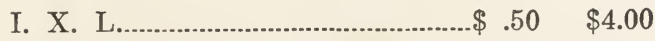

Texas Prolific .................................. $\quad .50 \quad 4.00$

Write for Prices for 100 or More 
م

PEARS-4 to $6 \mathrm{ft}$.-

Winter Bartlett ............................. $\quad .40 \quad 3.50$

Winter Nelis ................................... .40 3.50

3 to 4 Foot Trees.......................... .35 3.00

\section{PERSIMMONS-}

\section{Hachia}

Fuyu

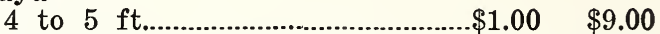

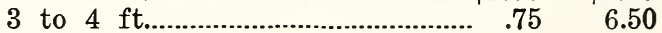

PLUMS-4 to $6 \mathrm{ft}$.-

Beauty …...................................... \$ .50 $\$ 4.00$

Santa Rosa ..................................... $\quad .50 \quad 4.00$

Burbank ........................................ .50 4.00

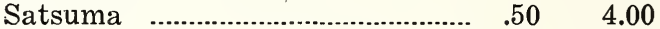

K Kellsie ................................................ $.50 \quad 4.00$

Wickson …........................................ $\quad .50 \quad 4.00$

Becky Smith ................................. $.50 \quad 4.00$

Blue Damson .................................. $\quad .50 \quad 4.00$

PRUNES-

Tragedy Prune ............................. $\$ .50 \quad \$ 4.00$

French Improved ......................... .50 4.00

CHERRIES-4 to $6 \mathrm{ft}$ - -

English Morello ............................ $\$ .60 \quad \$ 5.00$

Bing …............................................. ... $60 \quad 5.00$

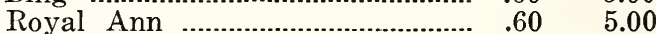

Black Tartarian ............................ $\quad .60 \quad 5.00$

\section{GRAPES-1 Year Roots-}

Thompson Seedless …..................\$ .20 $\$ 1.50$

White Malaga .................................. $\quad .20 \quad 1.50$

White Muscat ................................... $\quad .20 \quad 1.50$

Zinfandel ........................................ .20 $\quad .20$

Mission …........................................ $\quad .20 \quad 1.50$

Cornichon …...................................... $\quad .20 \quad 1.50$

Red Emperor ................................... . .20 1.50

$\begin{array}{llll}\text { Tokey …............................................ .20 } & .20\end{array}$

Concord ............................................ . .20 1.50

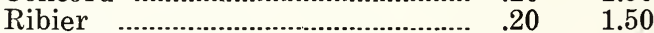

$\times \quad$ Elcante Bouche ................................ .20 $.20 \quad 1.50$

Black Morocco .............................. $\quad .20 \quad 1.50$

\section{WALNUTS AND PECANS}

\section{Placentia, Earhardt, Eureka}
10 to $12 \mathrm{ft}$
$\$ 1.60 \quad \$ 15.00$
8 to $10 \mathrm{ft}$
$1.25 \quad 10.00$
6 to $8 \mathrm{ft}$
7.5 ?
4 to $6 \mathrm{ft}$.
6.00

PECANS-5 to $7 \mathrm{ft}$.-

$\times$ Gower

Stuart

$\$ 2.50 \$ 20.00$

Success

20.00

20.00

Write for Prices for 100 or More

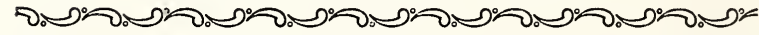




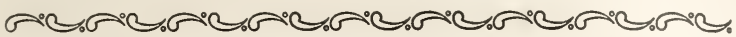

\section{RHUBARB ROOTS-}

Each $\quad 10 \quad 100$

Giant Winter, 1-yr.............\$ $.15 \quad \$ 1.00 \quad \$ 8.00$

Giant Winter, 2-yr............. $\quad .25 \quad 2.00 \quad 15.00$

Wagner Cherry,

$$
\begin{array}{llll}
\text { 2-yr., No. } 1 \text {............ } & .40 & 3.00 \\
\text { 2-yr., No. } & 2 \ldots \ldots \ldots \ldots . . . & .25 & 2.00
\end{array}
$$

Wagner Giant Seeds....................... $\$ 10.00$ per lb.

ASPARAGUS ROOTS-

$10 \quad 100 \quad 1000$

Mary Washington ............. $\$ .50 \quad \$ 1.00 \quad \$ 8.00$

\section{CURRANTS AND GOOSEBERRY}

CURRANTS-1 Year Roots-

Each Per 10

Cherry _.......................................... \$ .25 $\$ 2.00$

Wilder ............................................. .25 2.00

GOOSEBERRY-

Oregon Champion …..................... $\$ .25 \quad \$ 2.00$

\section{SPECIAL OFFERS-Postpaid-}

50 Mastodon Plants, and 4 Youngberry Tips, at

5 Logan Tips, 5 Youngberry Tips, 25 Mastodon

8 Youngberry Transplants, and 25

Champion Plants

25 Blakemore Plants, and 4 Youngberry

Transplants

2 Youngberry Transplants, and 25

Mastodon 1.00

\section{FERTILIZER}

Barnyard manure spread broadcast on the land after it is plowed and thoroughly disced in is the best thing you can do to add fertility to the land, and to get better growth on berry plants, and fruit. 


\title{
TIME OF SHIPMENT
}

We commence to fill Fall orders November 1st and ship all winter to sections suitable for Fall and Winter planting. For early Spring, March and April are the best months for berry planting. Our shipping season ends about May 1st. Freight and express are to be paid by purchaser.

\section{GENUINENESS}

We exercise great care to keep all varieties true to name, and will replace, on proper proof, all stock that may prove otherwise. We shall not be liable for any sum greater than that paid us for only such plants as may prove untrue.

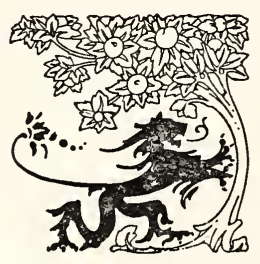

\section{Richards Nursery \& Berry Gardens}

\author{
CHINO, CALIFORNIA
}

